\title{
Traumatic Events and Substance Use Disorders in Adolescents
}

\author{
Lukas A. Basedow*, Sören Kuitunen-Paul, Veit Roessner and Yulia Golub \\ Department of Child and Adolescent Psychiatry, Faculty of Medicine, TU Dresden, Dresden, Germany
}

Objectives: Adolescents with substance use disorders (SUD) frequently report traumatic events (TEs) and symptoms of post-traumatic stress disorder (PTSD). This study aimed to assess whether lifetime prevalence rates of TEs and PTSD are related to SUD severity in adolescent psychiatric patients.

Methods: We analyzed $N=114$ self-reports of treatment-seeking German adolescents aged 12 to 18 years, who visited a specialized SUD outpatient unit. Standardized questionnaires were applied to assess SUD severity, the number of TEs and DSM-IV PTSD criteria.

Results: Patients fulfilling PTSD criteria (28\% of the total sample) had a higher Drug Use

OPEN ACCESS

Edited by:

David Belin,

University of Cambridge,

United Kingdom

Reviewed by:

Domenico De Berardis,

Azienda UsI Teramo, Italy

Mauro Ceccanti,

Sapienza University of Rome, Italy

*Correspondence:

Lukas A. Basedow

lukas.basedow@ukdd.de

Specialty section:

This article was submitted to Addictive Disorders,

a section of the journal

Frontiers in Psychiatry

Received: 30 December 2019 Accepted: 01 June 2020

Published: 18 June 2020

Citation:

Basedow LA, Kuitunen-Paul S, Roessner V and Golub Y (2020) Traumatic Events and Substance Use Disorders in Adolescents.

Front. Psychiatry 11:559. doi: 10.3389/fpsyt.2020.00559
Disorders Identification Test (DUDIT) score compared to non-PTSD patients with TEs $(p<.001)$, and compared to adolescents without TEs or PTSD $(p=.003)$. Additionally, SUD severity was positively associated with the number of TEs and the number of intrusion, hyperarousal, and avoidance symptoms (all $r=.33$ to.48, all $p<.01$ ).

Discussion: Adolescent patients with SUD reported 3-times higher rates of TEs, and a 5time higher prevalence of PTSD following TEs, than the general adolescent population. Adolescent SUD patients with PTSD reported more severe substance use problems than patients without PTSD-regardless of previous TEs. Longitudinal studies are needed in order to investigate the temporal relationship between TEs, PTSD and SUD.

Keywords: teenager, trauma, addiction, self-medication, traumatic experiences

\section{INTRODUCTION}

Adolescence, as defined by the World Health Organization, is a phase of life between ages 10 and 19 that marks the transition from childhood to adulthood $(1,2)$. Adolescents are "biologically wired" (3) to engage in risky and potentially harmful behavior including the use of psychoactive substances, which might lead to the development of a substance use disorder (SUD) (4). Moreover, the increase in risk behavior might lead to identification with substance-related subcultures (e.g. "psychonauts"), in which use of novel psychoactive substances (NPS) is widespread (5). In Germany, the lifetime prevalence rate of SUD in adolescents and young adults is $28.6 \%$ (6), with SUD being associated with worse performance in school, worse overall health, higher mortality rates and increased rates of comorbid psychiatric disorders such as post-traumatic stress disorder (PTSD) (7-9).

PTSD is an anxiety disorder that may develop subsequent to exposure to traumatic events (TEs), either experienced or witnessed. TEs are defined as experiences that involve actual or threatened 
death, serious injury, or threat to the physical integrity of oneself or others, and are responded to with intense fear, helplessness or horror (10). PTSD diagnosis requires an experience of a TE (Criterion A) that is followed by three major symptom clusters: intrusive recollections of the event (Criterion B), avoidance of event-related stimuli, or emotional numbing (Criterion $\mathrm{C}$ ) and hyperarousal (Criterion D).

Common developmental pathways for SUD and PTSD have been proposed, given the high rates of co-occurrence between the two disorders (11): Firstly, there is evidence from studies in adult patients that SUD and PTSD have common genetic and family environmental risk factors (12). Secondly, substance use in general is related to more frequent risk behavior (13), which increases the likelihood of experiencing TEs, and therefore developing PTSD $(14,15)$. For example, patients with a SUD are more likely to engage in other-directed than self-directed violence, increasing the risk of encountering TEs (16). Finally, the self-medication hypothesis derives from clinical observations that the specific actions or effects of each class of drugs relieve or change a range of painful affect states in patients with PTSD (11, $17,18)$.

Notably, the rate at which people are exposed to TEs peaks during adolescence (19) and this increased exposure is naturally associated with a higher rate of PTSD (14). In sum, adolescents typically engage in high-risk behavior thus increasing their risk for both substance use and TEs, which might lead to subsequent disorders, e.g. SUD and/or PTSD. There is a growing epidemiological and clinical literature documenting the frequent co-occurrence of SUD and PTSD in adolescents (20). Several studies indicate that between 20 and 54\% of North American adolescents with SUD also have co-occurring PTSD $(21,22)$. In German adolescents, SUD occurs at a rate of $30 \%$ in patients who fulfill PTSD criteria (23). Moreover, the severity of substance use (except for alcohol use) correlates positively with the number of PTSD symptoms in adolescents (24).

Previous research established the link between SUD and PTSD $(11,18,20,24)$. However, it remains to be explored if SUD severity is only increased in adolescents with co-occurring PTSD, or linked to the occurrence of TEs in general, providing an insight into the pathomechanisms of both disorders.

Here, we assess the prevalence of both lifetime TEs and PTSD according to DSM-IV criteria among German adolescents seeking treatment for a SUD and compare these prevalence rates with the general adolescent population. We compare three groups of SUD treatment-seeking adolescents: adolescents fulfilling DSM-IV PTSD diagnostic criteria ('PTSD' group), adolescents with a history of TEs but no PTSD ('TE' group) and adolescents with no TEs and no PTSD ('NoTE' group).

We hypothesize that (1) adolescents with a SUD present with higher rates of current PTSD and past TEs than the general adolescent population. We predict that (2) SUD severity will be higher in the PTSD and TE group than in the NoTE group and that (3) SUD severity is positively associated with the number of PTSD symptoms present in each symptom cluster.

\section{METHOD}

\section{Participants}

Between November 2017 and October 2019, $n=178$ treatmentseeking adolescents at an outpatient clinic for adolescent substance abuse participated in the study. $N=64$ adolescents (36\%) did not complete all necessary questionnaires and were therefore excluded. In the final sample of $n=114$ patients, the mean age was 15.8 years $(S D=1.3)$ with $43 \%(n=49)$ females, see Table 1. Secondary education levels were predominantly classified as low level (55\%), while household income was predominantly classified as medium level (57\%), see Table 1.

\section{Procedures}

Data collection was embedded into the standard diagnostic procedures at the outpatient clinic. Questionnaires were handed out to the patients and their legal guardians at the first consultation appointment in the outpatient department. The criteria for harmful use and dependence for all relevant psychoactive substances according to the ICD-10 guidelines (25), were assessed in a personal interview by a trained clinical psychologist. Study assessments took place before any intervention started. The study was conducted in accordance with the Declaration of Helsinki. Patients as well as legal guardians were informed about the projects thoroughly and comprehensively. Written informed consent was obtained from all legal guardians. All procedures of this study were approved by the Institutional Review Board of the University Hospital C. G. Carus Dresden (EK 66022018) and registered at clinicaltrials.gov (NCT03444974). No reimbursement was offered to patients.

\section{Measures}

\section{Traumatic Experiences, PTSD Symptoms and PTSD Diagnosis}

The University of California at Los Angeles Post Traumatic Stress Disorder Reaction Index for DSM-IV (UCLA PTSD (26) is a self-report instrument that screens for exposure to TEs, and assesses PTSD symptoms in school-age children and adolescents. It has been translated for, and used with German-speaking populations (27). The instrument consists of a trauma history section, in which patients indicate the TE that afflicts them the most and the traumatizing features of the event (Criterion A). The next section assesses the frequency of occurrence of PTSD symptoms during the past month (rated from $0=$ none of the time to $4=$ most of the time). The items map directly onto DSMIV intrusion/re-experience (Criterion B), avoidance (Criterion C), and hyperarousal (Criterion D) criteria. Scoring algorithms permit tabulation of UCLA PTSD total score, and A, B, C, and D subscale scores. The DSM-IV diagnosis PTSD is given when all four criteria (Criterion A, B, C, and D) are present (26). In the current sample, internal consistency was good for criterion A and $\mathrm{B}$ ( $\alpha=.83$ and.86, respectively), and acceptable for criterion $\mathrm{C}$ and $\mathrm{D}(\alpha=.73$ and.75, respectively). 
TABLE 1 | Sociodemographic characteristics of the complete sample, and the three subgroups.

\begin{tabular}{|c|c|c|c|c|c|c|c|}
\hline \multirow[t]{2}{*}{ Demographics } & \multirow{2}{*}{$\begin{array}{c}\text { Total } \\
(n=114)\end{array}$} & \multicolumn{3}{|c|}{ Analysis group } & \multicolumn{3}{|c|}{ Group comparison } \\
\hline & & $\begin{array}{c}\text { NoTE } \\
(n=35)\end{array}$ & $\begin{array}{c}T E \\
(n=47)\end{array}$ & $\begin{array}{l}\text { PTSD } \\
(n=32)\end{array}$ & $\begin{array}{l}\text { Test } \\
\text { statistic }(d f)\end{array}$ & $\mathbf{p}$ & $\begin{array}{l}\text { Effect } \\
\text { size }\end{array}$ \\
\hline Mean age $(S D)$ & $\begin{array}{l}15.8 \\
(1.3)\end{array}$ & $15.5(1.3)$ & $\begin{array}{l}16.0 \\
(1.3)\end{array}$ & $15.8(1.2)$ & $\begin{array}{c}F(113)= \\
1.32\end{array}$ & .271 & $\begin{array}{l}\eta_{\mathrm{p}}^{2}= \\
.023\end{array}$ \\
\hline Gender: & & & & & $\chi^{2}(2)=2.427$ & .297 & $\phi=.15$ \\
\hline Female (\%) & $49(43)$ & $12(34)$ & $27(57)$ & $17(53)$ & & & \\
\hline Male (\%) & $65(57)$ & $23(66)$ & $20(43)$ & $15(47)$ & & & \\
\hline Household income: \# of patients in category (\%), missing $n=60$ & $(n=54)$ & $(n=22)$ & $(n=21)$ & $(n=11)$ & $\chi^{2}(4)=1.525$ & .822 & .17 \\
\hline Low income & $9(17)$ & $4(18)$ & $4(19)$ & $1(9)$ & & & \\
\hline Middle income & $31(57)$ & $11(50)$ & $13(62)$ & $7(63)$ & & & \\
\hline High income & $14(26)$ & $7(32)$ & $4(19)$ & $3(25) 7$ & & & \\
\hline Educational level: \# of patients in category (\%), missing $n=38$ & $(n=76)$ & $(n=22)$ & $(n=34)$ & $(n=20)$ & $\chi^{2}(6)=4.773$ & .573 & .25 \\
\hline Low & $37(55)$ & $9(41)$ & $16(47)$ & $12(60)$ & & & \\
\hline Middle & $20(26)$ & $6(27)$ & $11(32)$ & $3(15)$ & & & \\
\hline High & $8(11)$ & $4(18)$ & $3(9)$ & $1(5)$ & & & \\
\hline Other & $11(14)$ & $3(14)$ & $4(12)$ & $4(20)$ & & & \\
\hline $\begin{array}{l}\text { Substance abuse: \# of patients presenting with harmful use or dependence per } \\
\text { substance (\%), missing } n=2\end{array}$ & $(n=112)$ & $(n=35)$ & $(n=47)$ & $(n=30)$ & & & \\
\hline Alcohol & $44(39)$ & 12 (34) & $23(49)$ & $9(30)$ & $\chi^{2}(2)=3.286$ & .193 & .17 \\
\hline Cannabis & $89(80)$ & $29(83)$ & $38(81)$ & $22(73)$ & $\chi^{2}(2)=0.993$ & .609 & .09 \\
\hline Stimulants (amphetamine, methamphetamine, or MDMA) & $49(44)$ & $13(37)$ & $18(38)$ & $18(60)$ & $\chi^{2}(2)=4.408$ & .110 & .20 \\
\hline
\end{tabular}

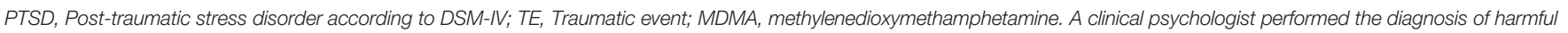

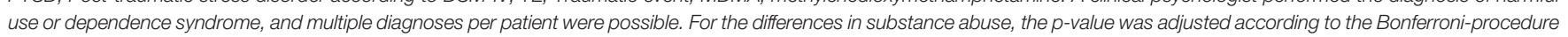
to $p<.02$.

\section{Substance Use and SUD Severity}

The Drug Use Disorders Identification Test [DUDIT; (28)] is a self-report instrument composed of 11 items identifying problems related to substance use. Scoring of the DUDIT is two-fold: items 1 to 9 are scored on a five-point Likert scale, while items 10 and 11 are scored on a three-point scale. The overall score is calculated by summing the scores on all items, with a maximum score of 44 . In the current sample, internal consistency of the DUDIT was good with $\alpha=.87$.

\section{SUD Diagnosis}

A clinical psychologist assessed criteria for harmful use and dependence syndrome for all relevant psychoactive substances according to ICD-10 (24) in a personal interview with the patients. The SUD diagnosis was established in a consensusbased procedure by interviewing psychologist and a boardcertified child and adolescent psychiatrist

\section{Sociodemographic Background}

Thirty-six generic questions were presented to the parents. We analyzed the questions indicating age in years, gender, and education level (low, medium, high) of the patient as well as yearly household income (low, medium, high). Adolescents' educational levels and parental income levels were assessed according to previously established criteria (29).

\section{Analyses}

All analyses were conducted with IBM SPSS Statistics 25.0. Based on UCLA PTSD results, patients were separated into three analysis groups: PTSD diagnosis ('PTSD' group), TE but no PTSD diagnosis ('TE' group), no TE and no PTSD diagnosis ('NoTE' group). To investigate the relationship between SUD and PTSD symptoms, Pearson's correlation coefficient $r$ was calculated between DUDIT score, the number of possible TEs, and the number of PTSD symptoms within each criterion cluster (Clusters A, B, C, and D). An analysis of variance (ANOVA) was performed to test for mean differences in DUDIT score between the three groups. To determine if the ANOVA is an appropriate procedure we checked the normality of the dependent variable (DUDIT score) by means of the Shapiro-Wilk test (30). Additionally, to check for homogeneity of variances, Levene's test was conducted. DUDIT score was normally distributed in the NoTE group $(W=.944 ; p=.072)$, the TE group $(W=.956$; $p=.075)$, and the PTSD group $(W=.952 ; p=.16)$. Further, Levene's test showed that the variance of DUDIT score was equal across groups, $F(2,111)=0.8, p=.452$. In cases were the $F$-test across groups was significant, post-hoc multiple comparisons using the Bonferroni correction were conducted to identify groups differing from each other regarding their mean DUDIT score. To detect demographic differences between the groups, $\chi^{2}$ tests were conducted. Level of significance was defined as $p<.05$ 
(two-tailed). Since patients could present with harmful use or dependence for multiple substances, multiple univariate $\chi^{2}$-tests were used to assess group differences in this variable. For this specific analysis, the level of significance was adapted according to the Bonferroni-procedure to $p<.05 / 3$. Effect sizes were classified according to Cohen (31) into small effects $(|r| \geq .10$, partial etasquare $\left.\eta_{\mathrm{p}}{ }^{2} \geq .01\right)$, medium effects $\left(|r| \geq .30, \eta_{\mathrm{p}}^{2} \geq .06\right)$, and large effects $\left(|r| \geq .50, \eta_{\mathrm{p}}^{2} \geq .14\right)$.

\section{RESULTS}

\section{Sociodemographic Characteristics and Substance Abuse}

The majority of patients reported enough symptoms to qualify for cannabis abuse (80\%), followed by alcohol abuse (39\%) and stimulant (amphetamine, methamphetamine, or MDMA) abuse (44\%), see Table 1. None of the patients reported use of NPS during the past 12 months. The three groups (PTSD, TE, and NoTE) did not differ in any of the assessed sociodemographic characteristics nor in their SUD diagnoses, see Table $\mathbf{1 .}$

\section{Prevalence Estimates}

The majority of patients (69\%) reported at least one lifetime TE. Figure 1 illustrates that "non-domestic violence" followed by "sexual abuse" were the most-prevalent TEs categories, whereas "war" and "medical treatment" represented the least-frequent categories. Over one third of the patients with TE (41\%) also fulfilled the DSM-IV diagnostic criteria for a PTSD according to the UCLA PTSD scale, resulting in a point prevalence of $28 \%$ for PTSD in the total sample.

\section{The Relationship Between TEs, PTSD Symptoms and SUD Severity}

The number of symptoms in each PTSD symptom cluster $(\mathrm{A}=$ number of traumatizing features of TEs, $\mathrm{B}=$ intrusion, $\mathrm{C}=$ avoidance, $\mathrm{D}=$ hyperarousal) correlated positively with each other (all $r=.23$ to .56 , all $p<.038$ ) and with DUDIT score (all $r=$ .33 to.48, all $p<.003$ ) with an exception of DUDIT score and Cluster A $(r=.20, p=.08)$. The correlations of DUDIT score and clusters B, C, and D can be classified as medium size effects ( $r=.30$ to .49), see Table 2.

\section{Group Differences in SUD Severity}

The three patient groups (PTSD, TE, NoTE) differed in their mean DUDIT score, $F(2,111)=7.86, p=.001, \eta_{\mathrm{p}}{ }^{2}=.124$. Posthoc multiple comparisons revealed that the PTSD group scored a significantly higher mean DUDIT score when compared to the TE group $(t(77)=3.812, p=.0008)$, and to the NoTE group $(t$ $(65)=3.33, p=.003)$. No difference was found between the TE and the NoTE group $(t(80)=.039, p=.969)$, see Figure 2 .

\section{DISCUSSION}

We investigated differences in SUD severity between three groups (PTSD, TE, NoTE) of treatment-seeking adolescents at a German outpatient clinic for adolescent substance abuse. Additionally, we assessed the past-month-prevalence of PTSD and lifetime-prevalence of TE. In accordance with our hypothesis as well as earlier studies, our results indicate that adolescents with SUD were more likely to report TEs [69\% vs. $21 \%(32)]$, more likely to suffer from PTSD following TEs [41\% vs. $8 \%$ (33)], and more likely to fulfill the diagnostic criteria for PTSD compared to the general adolescent population [28\% vs. $2 \%$ (32)]. Contrary to our hypothesis, only the PTSD group displayed more severe SUD symptoms than the TE group and the NoTE group. Additionally, we confirmed the hypothesis that SUD severity is positively associated with the number of hyperarousal, intrusion, and avoidance symptoms.

We were not able to delineate the reason for the positive relationship between the number of PTSD symptoms and SUD severity. Patients with stronger PTSD symptoms might selfmedicate more heavily with psychotropic substances, and

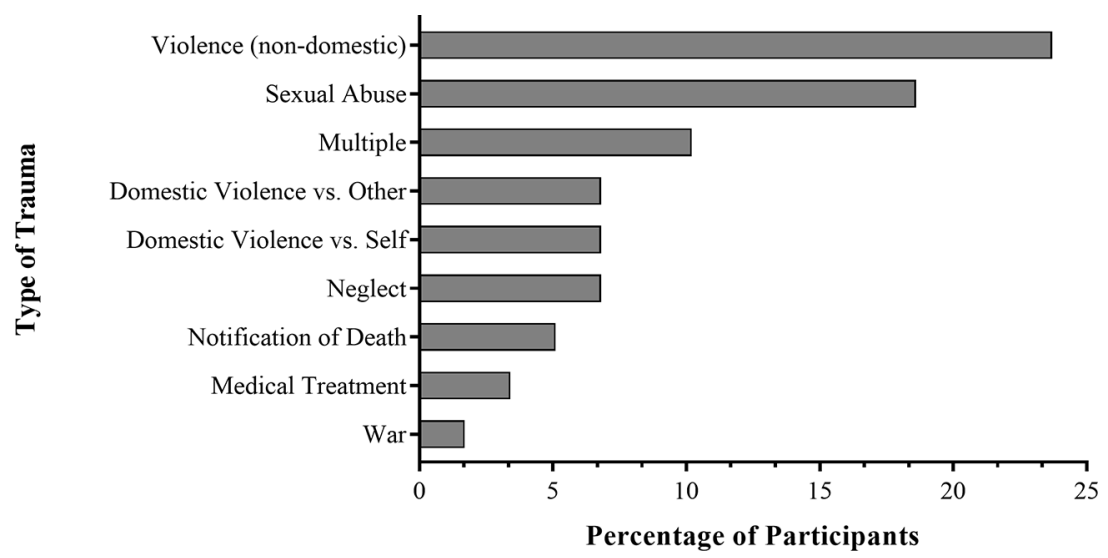

FIGURE 1 | The percentage of patients $(n=59)$ that described a particular type of event as their most traumatizing experience. Total $n=114$, with $n=35$ reporting no traumatic experiences, and $n=20$ not clearly identifying the most traumatizing experience. 
TABLE 2 | Bivariate Pearson correlation coefficients between DUDIT score, the number of Traumatic events (TE), and the number of symptoms present in Clusters A, $\mathrm{B}, \mathrm{C}$, and D of the UCLA PTSD Questionnaire.

\begin{tabular}{|c|c|c|c|c|c|c|}
\hline & DUDIT score & Number of TE & Sum Cluster A & Sum Cluster B & Sum Cluster C & Sum Cluster D \\
\hline DUDIT score & - & $.21^{*}$ & .20 & $.33^{\star \star}$ & $.35^{\star \star}$ & $.48^{* \star}$ \\
\hline Number of TE & $.21^{*}$ & - & .13 & $.34^{\star \star}$ & $.27^{\star}$ & .16 \\
\hline Sum Cluster A & .20 & .13 & - & $.45^{\star \star}$ & $.27^{\star}$ & $.23^{\star}$ \\
\hline Sum Cluster B & $.33^{\star \star}$ & $.34^{\star \star}$ & $.45^{\star \star}$ & - & $.56^{\star \star}$ & $.54^{\star \star}$ \\
\hline Sum Cluster C & $.35^{\star \star}$ & $.27^{*}$ & $.27^{\star}$ & $.56^{\star \star}$ & - & $.47^{\star \star}$ \\
\hline Sum Cluster D & $.48^{\star \star}$ & .16 & $.23^{\star}$ & $.54^{\star \star}$ & $.47^{\star \star}$ & - \\
\hline
\end{tabular}

DUDIT, Drug use disorders identification test; PTSD, Post-traumatic stress disorder according to DSM-IV. Cluster A = Traumatizing qualities of event. Cluster B = Intrusion. Cluster C = Avoidance. Cluster D = Hyperarousal. SUD, substance use disorder according to ICD-10, including substance abuse and substance dependence; TE, Traumatic event. ${ }^{*} P<0.05$,

${ }^{* *} p<0.01$. The number of cases per cell varied due to presence of a PTSD between $n=79$ and 114 .

therefore develop more SUD symptoms (11, 17, 18). Alternatively, when functional substance use develops into a SUD, PTSD symptoms might increase as a results of reduced overall mental health (34). Additionally, the development of SUD-related withdrawal symptoms, such as anxiety or depression, might lead to stronger PTSD symptoms (34). Consequently, self-medication of PTSD symptoms with psychotropic substances should be seen as a risk factor, not only for a subsequent SUD, but also for an increase of PTSD symptoms later on. It remains to be explored, if patients with more severe PTSD engage in more substance use, if patients with heavier substance use are more vulnerable to develop PTSD following TEs, or whether a more complex bidirectional relationship exists. To assess these complex relationships, longitudinal studies are needed that frequently assess occurrence of TEs, PTSD symptoms, substance use, and SUD symptoms.

Our finding that TEs alone are not associated with higher SUD severity is supported by research examining the relationship between PTSD and SUD in adolescents and adults (35-36). Reed et al. (37) found that in adolescents, the presence of PTSD but not TEs without PTSD is associated with a higher risk for future substance abuse or dependence. Furthermore, Driessen et al. (35) observed that a PTSD diagnosis but not the number of TEs correlates with SUD severity. Finally, Kok et al.
(36) reported, that trauma-related variables do not add information about variation in drug use severity compared to PTSD symptoms. This line of research indicates that TEs are unrelated to SUD severity and the development of SUD symptoms.

Previous studies with adult SUD patients reported similar results to ours, insofar as all three symptom clusters correlated positively with SUD severity $(36,38)$. However, research in adolescents indicated only avoidance symptoms being positively associated with SUD severity, whereas hyperarousal symptoms were negatively correlated with SUD severity, and intrusion symptoms were not related to SUD severity at all (24). These conflicting results might be explained by the difference in sample selection. Specifically, we included patients with a SUD regardless of substance, whereas Donbaek et al. (24) excluded patients with an alcohol use disorder (AUD). Thus, the substance of use could be relevant for the relationship between SUD and PTSD symptoms. Indeed, a relationship between the use of specific substances and PTSD symptoms has been observed to exist in adults and adolescents. Explicitly it has been reported that the number of avoidance symptoms is higher in people who use alcohol $(11,38)$, sedatives, gamma-Hydroxybutyric acid (GHB), opioids (39), and heroin specifically (40). Moreover, people with an alcohol (38) or opioid (39) use disorder present with more hyperarousal symptoms than people who do not.

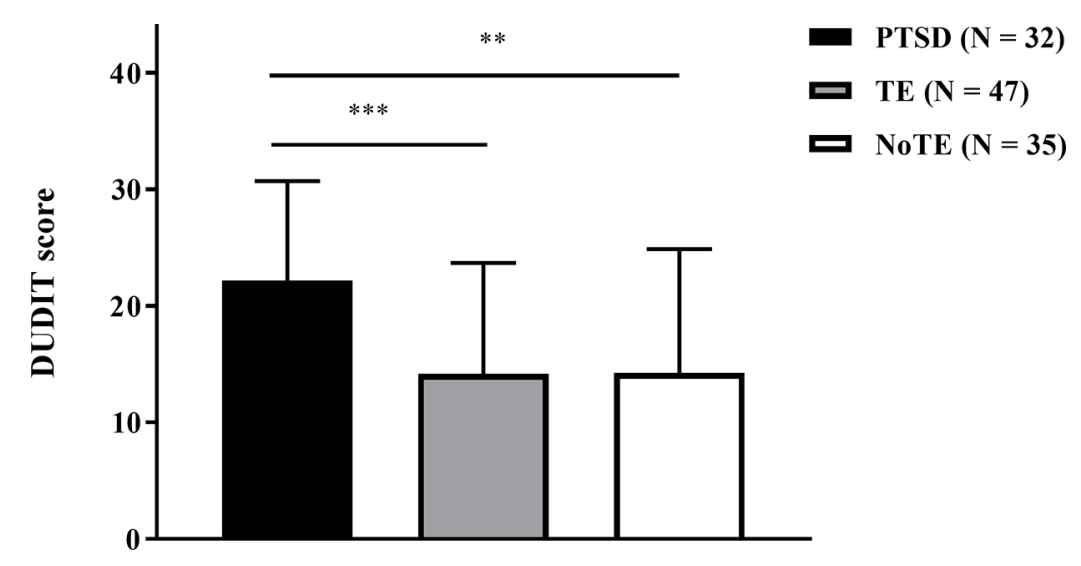

FIGURE 2 | The mean DUDIT score for the PTSD group $(n=32)$, the TE group $(n=47)$, and the NoTE group $(n=35)$. Mean differences were calculated through post-hoc multiple comparisons using the Bonferroni correction $\left({ }^{* *} p<0.01,{ }^{\star \star *} p<0.001\right)$. 
Finally, Khoury et al. (38) and Avant et al. (39) report that the use of cannabis, cocaine, sedatives, and GHB is also related to more intrusion symptoms. While the use of specific substances does not seem to be related to specific symptom clusters, recent findings $(11,24,38-40)$ confirm our results, that a higher level of substance use across substances is generally related to a higher number of PTSD symptoms across clusters.

\section{Limitations}

Our sample was limited to treatment-seeking adolescents with a SUD. Therefore, future research could investigate whether the presence of TE or PTSD influences the intensity of recreational substance use as well or if this influence is limited to SUD severity.

Our assessment of TEs and PTSD was based on self-report measures, which might be biased in the sense that patients might not recall all of their TEs. Additionally, all self-report instruments potentially suffer from response bias. Examples include misunderstanding of the items or a social-desirability bias (41). Furthermore, self-reports seem to underestimate trauma-specific cognitions, like intrusion symptoms (42). In future research it might be useful to use additional instruments such as standardized interviews to gain a more accurate picture of the PTSD symptoms clusters and number of TEs.

Furthermore, because we did not collect detailed data on substance use, we could not investigate the relationship between the use of specific substances and the number of PTSD symptoms in each cluster. Additionally, we took no measures to verify self-reports of substance use via biological measures. Therefore, even though none of the patients reported use of NPS, they might have unwillingly ingested them instead of a substance they were sold (e.g. NPS in an ecstasy pill).

Finally, our cross-sectional study lacks a possibility to investigate the question of timing and causality concerning PTSD and SUD development. A prospective longitudinal study design is necessary in order to answer the question, which symptoms or disorders develop first, and if TEs precede substance use or vice versa. This line of research would help to clarify the hypotheses concerning the relationship between the two disorders. For example, the finding that substance use and SUD symptoms generally appear after a TE would support the self-medication hypothesis.

\section{REFERENCES}

1. Plummer ML, Baltag V, Strong K, Dick B, Ross DA. Global Accelerated Action for the Health of Adolescents (AA-HA!): guidance to support country implementation. World Health Organization (2017).

2. Waltereit R, Uhlmann A, Roessner V. Adolescent psychiatry-from the viewpoint of a child and adolescent psychiatrist. Eur Child Adolesc Psychiatry (2018) 27:1383-5. doi: 10.1007/s00787-018-1231-z

3. Kipping RR, Campbell RM, MacArthur GJ, Gunnell DJ, Hickman M. Multiple risk behaviour in adolescence. J Public Health (2012) 34:i1-2. doi: 10.1093/pubmed/fdr122

4. Behrendt S, Wittchen H-U, Höfler M, Lieb R, Beesdo K. Transitions from first substance use to substance use disorders in adolescence: is early onset associated with a rapid escalation? Drug Alcohol Depend (2009) 99:68-78. doi: 10.1016/j.drugalcdep.2008.06.014

\section{Conclusion}

Adolescents with SUD reported 3-time higher rates of TEs, and a 5-time higher prevalence of PTSD following TEs, than the general adolescent population. SUD was more severe in adolescents with PTSD than in adolescents without TEs or with TE anamnesis but no PTSD symptoms. Finally, the level of SUD severity was positively correlated with the number of PTSD-related intrusion, avoidance, and hyperarousal symptoms.

\section{DATA AVAILABILITY STATEMENT}

The datasets generated for this study are available on request to the corresponding author.

\section{ETHICS STATEMENT}

The studies involving human participants were reviewed and approved by Institutional Review Board of the University Hospital C. G. Carus, Dresden (EK 66022018). Written informed consent to participate in this study was provided by the participants' legal guardian/next of kin.

\section{AUTHOR CONTRIBUTIONS}

LB analyzed the data and wrote the manuscript. SK-P participated in writing the manuscript and data analysis, and contributed to the discussion. VR participated in writing the manuscript and contributed to discussion. YG designed the study, participated in writing the manuscript, and contributed to discussion.

\section{FUNDING}

The Sächsische Aufbaubank -Förderbank-, (grant 100362999 to YG), funded this study. Open access funding by the Publication Fund of the TU Dresden.

5. Orsolini L, Ciccarese M, Papanti D, De Berardis D, Guirguis A, Corkery JM et al. Psychedelic Fauna for Psychonaut Hunters: A Mini-Review. Front Psychiatry (2018) 9:153. doi: 10.3389/fpsyt.2018.00153

6. Perkonigg, Pfister H, Höfler M, Fröhlich C, Zimmermann P, Lieb R, et al. Substance Use and Substance Use Disorders in a Community Sample of Adolescents and Young Adults: Incidence, Age Effects and Patterns of Use. Eur Addict Res (2006) 12:187-96. doi: 10.1159/000094421

7. Lindblad R, Hu L, Oden N, Wakim P, Rosa C, VanVeldhuisen P. Mortality Rates among Substance Use Disorder Participants in Clinical Trials: Pooled Analysis of Twenty-two Clinical Trials within the National Drug Abuse Treatment Clinical Trials Network. J Subst Abuse Treat (2016) 70:73-80. doi: 10.1016/j.jsat.2016.08.010

8. Rattermann MJ. Measuring the impact of substance abuse on student academic achievement and academic growth. Adv Sch Ment Health Promot (2014) 7:123-35. doi: 10.1080/1754730X.2014.888225 
9. Schulte MT, Hser Y-I. Substance Use and Associated Health Conditions throughout the Lifespan. Public Health Rev (2014) 35:2. doi: 10.1007/ BF03391702

10. American Psychiatric Association. Diagnostic and Statistical Manual of Mental Disorders. 4th ed. Washington, D.C.: American Psychiatric Association (2000). doi: 10.1176/appi.books.9780890425596

11. Dworkin ER, Wanklyn S, Stasiewicz PR, Coffey SF. PTSD symptom presentation among people with alcohol and drug use disorders: Comparisons by substance of abuse. Addict Behav (2018) 76:188-94. doi: 10.1016/j.addbeh.2017.08.019

12. Xian H, Chantarujikapong SI, Scherrer JF, Eisen SA, Lyons MJ, Goldberg J, et al. Genetic and environmental influences on posttraumatic stress disorder, alcohol and drug dependence in twin pairs. Drug Alcohol Depend (2000) 61:95-102. doi: 10.1016/S0376-8716(00)00127-7

13. Baskin-Sommers A, Sommers I. The co-occurrence of substance use and highrisk behaviors. J Adolesc Health (2006) 38:609-11. doi: 10.1016/ j.jadohealth.2005.07.010

14. Glaesmer H, Matern B, Rief W, Kuwert P, Braehler E. Traumatisierung und posttraumatische Belastungsstörungen. Nervenarzt (2015) 86:800-6. doi: 10.1007/s00115-014-4235-Z

15. Strom TQ, Leskela J, James LM, Thuras PD, Voller E, Weigel R, et al. An exploratory examination of risk-taking behavior and PTSD symptom severity in a veteran sample. Mil Med (2012) 177:390-6. doi: 10.7205/MILMED-D-1100133

16. Harford TC, Yi H, Grant BF. Other- and Self-Directed Forms of Violence and Their Relationships to DSM-IV Substance Use and Other Psychiatric Disorders in a National Survey of Adults. Compr Psychiatry (2013) 54:7319. doi: $10.1016 /$ j.comppsych.2013.02.003

17. Khantzian EJ. The self-medication hypothesis of addictive disorders: focus on heroin and cocaine dependence. Am J Psychiatry (1985) 142:1259-64. doi: 10.1176/ajp.142.11.1259

18. McCauley JL, Killeen T, Gros DF, Brady KT, Back SE. Posttraumatic Stress Disorder and Co-Occurring Substance Use Disorders: Advances in Assessment and Treatment. Clin Psychol Publ Div Clin Psychol Am Psychol Assoc (2012) 19:3. doi: 10.1111/cpsp.12006

19. Breslau N. The epidemiology of trauma, PTSD, and other posttrauma disorders. Trauma Violence Abuse (2009) 10:198-210. doi: 10.1177/ 1524838009334448

20. Brady KT, Back SE, Coffey SF. Substance Abuse and Posttraumatic Stress Disorder. Curr Dir Psychol Sci (2004) 13:206-9. doi: 10.1111/j.09637214.2004.00309.x

21. Turner WC, Muck RD, Muck RJ, Stephens RL, Sukumar B. Co-occurring disorders in the adolescent mental health and substance abuse treatment systems. J Psychoactive Drugs (2004) 36:455-62. doi: 10.1080/ 02791072.2004.10524428

22. Williams JK, Smith DC, An H, Hall JA. Clinical outcomes of traumatized youth in adolescent substance abuse treatment: a longitudinal multisite study. J Psychoactive Drugs (2008) 40:77-84. doi: 10.1080/02791072.2008.10399763

23. Essau, Conradt J, Petermann F. Häufigkeit der Posttraumatischen Belastungsstörung bei Jugendlichen: Ergebnisse der Bremer Jugendstudie. $Z$ Für Kinder- Jugendpsychiatrie Psychother (1999) 27:37-45. doi: 10.1024// 1422-4917.27.1.37

24. Donbaek DF, Elklit A, Pedersen MU. Post-traumatic stress disorder symptom clusters predicting substance abuse in adolescents. Ment Health Subst Use (2014) 7:299-314. doi: 10.1080/17523281.2013.873071

25. World Health Organization. The ICD-10 classification of mental and behavioural disorders: clinical descriptions and diagnostic guidelines. Geneva: World Health Organization (2009).

26. Steinberg AM, Brymer MJ, Decker KB, Pynoos RS. The University of California at Los Angeles Post-traumatic Stress Disorder Reaction Index. Curr Psychiatry Rep (2004) 6:96-100. doi: 10.1007/s11920-004-0048-2

27. Ruf M, Schauer M, Elbert T. (2011). UPID : UCLA PTSD Index for DSM IV (Child version, revision 1, deutsche Fassung). Hogrefe

28. Voluse AC, Gioia CJ, Sobell LC, Dum M, Sobell MB, Simco ER. Psychometric properties of the Drug Use Disorders Identification Test (DUDIT) with substance abusers in outpatient and residential treatment. Addict Behav (2012) 37:36-41. doi: 10.1016/j.addbeh.2011.07.030

29. Lampert T, Hoebel J, Kuntz B, Müters S, Kroll LE. Messung des sozioökonomischen Status und des subjektiven sozialen Status in KiGGS Welle 2. RKI-Bib1 Robert Koch-Inst (2018) 3(1):44-61 doi: 10.17886/rkigbe-2018-016

30. Howell DC. Statistical methods for psychology. Wadsworth: Cengage Learning (2002).

31. Cohen J. Statistical power analysis for the behavioral sciences. 2. ed. New York, NY: Psychology Press (1988).

32. Essau, Conradt J. Phil. Frequency, Comorbidity, and Psychosocial Impairment of Anxiety Disorders in German Adolescents. J Anxiety Disord (2000) 14:263-79. doi: 10.1016/S0887-6185(99)00039-0

33. Perkonigg, Kessler RC, Storz S, Wittchen H-U. Traumatic events and posttraumatic stress disorder in the community: prevalence,risk factors and comorbidity. Acta Psychiatr Scand (2000) 101:46-59. doi: 10.1034/j.16000447.2000.101001046.x

34. Simmons S, Suárez L. Substance Abuse and Trauma. Child Adolesc Psychiatr Clin N Am (2016) 25:723-34. doi: 10.1016/j.chc.2016.05.006

35. Driessen M, Schulte S, Luedecke C, Schaefer I, Sutmann F, Ohlmeier M, et al. Trauma and PTSD in patients with alcohol, drug, or dual dependence: a multi-center study. Alcohol Clin Exp Res (2008) 32:481-8. doi: 10.1111/j.15300277.2007.00591.x

36. Kok T, de Haan H, van der Meer M, Najavits L, de Jong C. Assessing traumatic experiences in screening for PTSD in substance use disorder patients: What is the gain in addition to PTSD symptoms? Psychiatry Res (2015) 226:328-32. doi: 10.1016/j.psychres.2015.01.014

37. Reed PL, Anthony JC, Breslau N. Incidence of Drug Problems in Young Adults Exposed to Trauma and Posttraumatic Stress Disorder: Do Early Life Experiences and Predispositions Matter? Arch Gen Psychiatry (2007) 64:1435. doi: 10.1001/archpsyc.64.12.1435

38. Khoury L, Tang YL, Bradley B, Cubells JF, Ressler KJ. Substance use, childhood traumatic experience, and Posttraumatic Stress Disorder in an urban civilian population. Depress Anxiety (2010) 27:1077-86. doi: 10.1002/ da. 20751

39. Avant EM, Davis JL, Cranston CC. Posttraumatic Stress Symptom Clusters, Trauma History, and Substance Use among College Students. J Aggress Maltreatment Trauma (2011) 20:539-55. doi: 10.1080/10926771.2011.588153

40. Tull MT, Gratz KL, Aklin WM, Lejuez CW. A Preliminary Examination of the Relationships between Posttraumatic Stress Symptoms and Crack/Cocaine, Heroin, and Alcohol Dependence. J Anxiety Disord (2010) 24:55-62. doi: 10.1016/j.janxdis.2009.08.006

41. Rosenman R, Tennekoon V, Hill LG. Measuring bias in self-reported data. Int J Behav Healthc Res (2011) 2:320-32. doi: 10.1504/IJBHR.2011.043414

42. Takarangi MKT, Strange D, Lindsay DS. Self-report may underestimate trauma intrusions. Conscious Cognit (2014) 27:297-305. doi: 10.1016/ j.concog.2014.06.002

Conflict of Interest: SK-P received honoraria/fees during the past 12 months: author fees from a publisher of medical books (Mabuse Verlag), and honoraria for one speech from a group of companies (AbbVie Deutschland, Almirall Hermal, Belano medical, Celgene, Janssen-Cilag, LEO Pharma, Lilly Deutschland, Novartis Pharma, Pfizer Pharma, UCB Pharma).

The remaining authors declare that the research was conducted in the absence of any commercial or financial relationships that could be construed as a potential conflict of interest.

Copyright (c) 2020 Basedow, Kuitunen-Paul, Roessner and Golub. This is an openaccess article distributed under the terms of the Creative Commons Attribution License (CC BY). The use, distribution or reproduction in other forums is permitted, provided the original author(s) and the copyright owner(s) are credited and that the original publication in this journal is cited, in accordance with accepted academic practice. No use, distribution or reproduction is permitted which does not comply with these terms. 